\title{
Keeping our eyes on CRISPR: the "Atlas" of gene editing
}

\author{
William Wang • Jiayuan Hou • Nannan Zheng • \\ Xiangdong Wang • Jiaqiang Zhang
}

Received: 5 May 2019 / Accepted: 16 May 2019/Published online: 5 June 2019

(C) Springer Nature B.V. 2019

CRISPR technology has evolved from a promising gene editing tool to revolutionizing the field of molecular biology by providing scientists with a tool that can target desired locations in the genome. It is almost inevitable that CRISPR will find usage clinically due to its rapid improvement in sensitivity, accuracy, reliability, and efficiency of nucleic acid measurement. Since 2012, there has been a continuously increasing amount of publications related to CRISPR in "PubMed." It is not an exaggeration to say that CRISPR will be pivotal in the future development and implementation of gene editing technology and could perhaps even play a similar role to "Atlas" in Greek mythology, with the weight of the biological medicine on its shoulders. However, that reality is still years away and important issues still remain, including the urgent needs to develop an accurate and easy methodology to dynamically monitor the

William Wang, Jiayuan Hou and Nannan Zheng contribute to this article equally as the first author

W. Wang $\cdot$ J. Hou $\cdot$ N. Zheng $\cdot$ X. Wang $(\bowtie)$

Zhongshan Hospital Institute for Clinical Science, Shanghai Institute of Clinical Bioinformatics, Shanghai Engineering Research for AI Technology for Cardiopulmonary Diseases, Center for Tumor Diagnosis and Therapy, Jinshan Hospital, Shanghai Medical College, Fudan University, Shanghai, China e-mail: xiangdong.wang@ clintransmed.org

J. Zhang $(\bowtie)$

Department of Anaesthesiology, Centre for Clinical Single Cell Biomedicine, Henan provincial People's Hospital, People's Hospital of Zhengzhou University, Zhengzhou, China e-mail: jqzhang@henu.edu.cn changes of gene sequencing before and after gene editing, to have clear and restricted regulations of gene editing application, to establish legal regulations and bioethics of gene modification practice, and how to optimize the procedures of in vivo gene editing.

One way to achieve a solution to these issues is to improve the sensitivity, accuracy, reliability, and efficiency of nucleic acid measurement, making it possible for clinical application. The type II CRISPR-Cas9 system derived from Streptococcus pyogenes (SpCas9) is most used due to the simplicity of $5^{\prime}-\mathrm{NGG}-3^{\prime}$ protospacer adjacent motif sequence requirement, while other CRISPR systems also show promising effects, e.g., Cas12 from Lachnospiraceae (LbCas12) and Acidaminococcus sp. (AsCas12) and Cas13 from Leptotrichia wadei (LwCas13a) (Shmakov et al. 2017; Yamano et al. 2016; Zetsche et al. 2015). Although Cas12a and Cas9 rely on protospacer adjacent motif recognition to target DNA, Cas9 cleaves the target strand and nontarget strand DNA via HNH and RuvC domains, while Cas 12 a cleaves dsDNA via utilizing a RuvC catalytic domain as a target for RNA-directed cleavage (Swarts et al. 2017; Zetsche et al. 2015). Cas12a enzymes independently catalyze guide RNA (crRNA) maturation and recognizes the 5'-TTTV-3' PAM (V indicates either A, C, or G) upstream of the protospacer sequence (Zetsche et al. 2015). Cas13 variants function via targeting single-stranded RNA and are used as a base to develop RNA-guided technology to target RNA, perform modules and perturbations, tag for localization, and monitor or capture transcripts in cells (Abudayyeh et al. 2016). 
The quality of measurements has been improved and modified rapidly. Gootenberg et al. (2017) developed a detection platform named Specific High-sensitivity Enzymatic Reporter UnLOCKing (SHERLOCK) which combines the usage of isothermal pre-amplification and Cas13a to detect nucleotides at an attomole level for both viruses and bacteria. An appropriately designed Cas13a guide RNA in conjunction with SHERLOCK can identify various strains of the same virus such as synthetic Zika and Dengue viruses or polymorphisms such as mutations in EGFR. Subsequently, SHERLOCK was recently updated to SHERLOCKv2 which is based upon four-channel simple multiplexing with orthogonal and nucleic acid sequences of LwaCas13a, PsmCas13b, CcaCas13b, and AsCas12a. Through these channels, SHERLOCKv2 could detect a multitude of DNA and RNA sequences in a solitary sample (Gootenberg et al. 2018). In contrast, Chen et al. (2018) found that Cas12a could function as a potential nucleic acid detection platform and developed a DNA Endonuclease-Targeted CRISPR Trans Reporter (DETECTR). Similarly, this platform utilizes isothermal amplification and Cas12a ssDNase activation for attomole DNA detection and were able to differentiate between two human papillomavirus strains (16 and 18) with high accuracy.

A number of emerging opportunities appear in the process to translate those methodologies into clinical performance. Of those, a practical challenge is interpreting the exact meaning behind changes of nucleic acids in the circulation without cancer information as nuclear acids can be either be driven from multi-organs/tissues, cells, and diseases, or from various origins of biological processes, e.g., mutations, differentiations, evolutions, methylation, and repair. The designed and selected target panels of nucleic acids from a disease may provide a non-invasive diagnostic tool for dynamical monitoring of pre- or post-on-target and off-target, especially the liquid biopsies of cancer. It is questioned how the disease-specific nucleic acids can fit the individual profiles and phenotypes of each patient during early diagnosis, large-scale screening, dynamical monitoring, and response to therapy (Wang and Wang 2019).

The aim of CRISPR drives is to tip the scales in inheritance patterns and increase the chance of a desired sequence of DNA being inherited in offspring, and ultimately affecting the local population and other species that interact with them. The US Defense Advanced Research Projects Agency (DARPA) has considered it to be a "national security issue" and have already highlighted its potential in targeting malaria transmission to eliminate malaria (Callaway 2017; Gantz et al. 2015). However, key concerns remain unanswered including unplanned genetic alterations in an entire species and laboratory safety protocols to prevent unintended gene drive spread which could cause catastrophic damage for the natural ecosystem. Two types of gene drives could potentially address the later issue in which synthetic drives target engineered genomic sites absent in wild species, or split drives without the endonuclease require an engineered site present on an unlinked site (Champer et al. 2019). Those two types of drives into Drosophila melanogaster and targeting enhanced green fluorescent protein and X-linked genes could show a similar behavior to normal drives and importantly were found to be viable in population-suppression drives as well.

Risks of off-target effects of CRISPR re-entered the spotlight when a publication came under heavy criticism and skepticism for claiming that CRISPR-Cas9 nuclease could induce more than a thousand unintended offtarget alterations at genomic loci when compared to a control group (Schaefer et al. 2017). The paper was ultimately retracted after several correspondences which highlighted deficiencies in the study including a poor experimental design, conclusion not correlating with the data, insignificant sample size, and failing to consider the possibility of the variations existing prior to nucleotide treatment. Implications of off-target effects with the commonly used wild-type SpCas9 has been observed in studies, and variations to SpCas9 have been suggested to reduce off-target effects while maintaining reasonable on-target effects such as SpCas9-High fidelity no. 1 (SpCas9-HF1) and seem to be reasonable strategies for addressing potential off-target effects (Kleinstiver et al. 2016). Heavy emphasis by scientists have been to address off-target effects, while recent studies also discuss the potential of undesired on-target effects including deletions of kilobases, crossover events, and inversions which result in neoplasia in transcriptionally active locus (Kosicki et al. 2018). Although concerns have also been raised relating to p53/TP53-dependent toxicity to Cas9 in human-induced pluripotent stem cells, it remains to serve as a caution to ensure functional p53 gene expressions in Cas9 edited cells (Ihry et al. 2018).

The ethical dilemmas CRISPR faces have been mentioned previously in multiple publications (Fang and Wang 2016; Organizing Committee for the 
International Summit on Human Gene Editing 2015; Wang et al. 2018) and, however, were centered around the importance of cautious experimentation and suitable framework as well as the need for international guidelines regarding gene editing in human germlines. The ethical aspect of gene editing has come under public scrutiny when gene-edited twin girls were born in China in November 2018. A global moratorium has been issued to ensure all clinical uses of human germline editing and emphasize the importance of international frameworks and regulations (Evitt et al. 2015). The framework suggested includes a fixed period of a ban of all clinical activities involving gene editing, the requirement of a public notice prior to application with a careful evaluation of both the suitability and justification, and the creation of a coordinating body to implement and support the framework. The moratorium proposed would significantly reduce progression of gene editing in humans and could result in stagnation of gene editing for an indefinite amount of time. The risk involved in implementing uncontrolled gene changes in germlines could result in disastrous results and importantly could pose personal danger to the patient as highlighted in the case where Dr. He through inactivation of CCR5 has put the patient at increased risk of viral infections including influenza and West Nile virus. With our current understanding, it is near to impossible to predict the potential effects with gene modifications in germlines and their impact on future generations and caution must be used to proceed.

With phase 1 of clinical trials being green-lit in both the USA and Europe for CRISPR technology, it is undisputable that CRISPR will become a key technology for biological and scientific research. However, the future CRISPR faces is not without its perils and hurdles and a variety of challenges remain to be overcome before clinical application. The social and ethical aspects of gene editing are becoming key issues that need to be addressed with the ever increasing social and mass media interest in gene editing. The importance of international frameworks to regulate future germline experiments cannot be understated and to alleviate public concerns. Furthermore, additional extensive studies regarding both on-target and off-target effects should be carried out to further clarify potential implications of immunogenicity of Cas 9 proteins due to repeated administrations, improve efficiency levels for transferring and editing genes, and improve genotoxicity from vectors. However, it is no doubt that CRISPR will become an emerging discipline in providing better healthcare and improving human health and a ready tool for clinical application in near future.

\section{References}

Abudayyeh OO, Gootenberg JS, Konermann S, Joung J, Slaymaker IM, Cox DBT, et al. C2c2 is a single-component programmable RNA-guided RNA-targeting CRISPR effector. Science. 2016;353:aaf5573. https://doi.org/10.1126 /science.aaf5573.

Callaway E. US defence agencies grapple with gene drives. Nature. 2017;547:388-9. https://doi.org/10.1038 /nature.2017.22345.

Champer J, Chung J, Lee YL, Liu C, Yang E, Wen Z, et al. Molecular safeguarding of CRISPR gene drive experiments. Elife. 2019;8. https://doi.org/10.7554/eLife.41439.

Chen JS, Ma E, Harrington LB, Da Costa M, Tian X, Palefsky JM, et al. CRISPR-Cas12a target binding unleashes indiscriminate single-stranded DNase activity. Science. 2018;360:4369. https://doi.org/10.1126/science.aar6245.

Evitt NH, Mascharak S, Altman RB. Human germline CRISPRCas modification: toward a regulatory framework. Am J Bioeth. 2015;15:25-9. https://doi.org/10.1080 /15265161.2015.1104160.

Fang H, Wang W. Could CRISPR be the solution for gene editing's Gordian knot? Cell Biol Toxicol. 2016;32:465-7. https://doi.org/10.1007/s10565-016-9359-4.

Gantz VM, Jasinskiene N, Tatarenkova O, Fazekas A, Macias VM, Bier E, et al. Highly efficient Cas9-mediated gene drive for population modification of the malaria vector mosquito Anopheles stephensi. Proc Natl Acad Sci U S A. 2015;112: E6736-43. https://doi.org/10.1073/pnas.1521077112.

Gootenberg JS, Abudayyeh OO, Kellner MJ, Joung J, Collins JJ, Zhang F. Multiplexed and portable nucleic acid detection platform with Cas13, Cas12a, and Csm6. Science. 2018;360:439-44. https://doi.org/10.1126/science.aaq0179.

Gootenberg JS, Abudayyeh OO, Lee JW, Essletzbichler P, Dy AJ, Joung J, et al. Nucleic acid detection with CRISPR-Cas13a/ C2c2. Science. 2017;356:438-42. https://doi.org/10.1126 /science.aam9321.

Ihry RJ, Worringer KA, Salick MR, Frias E, Ho D, Theriault K, et al. p53 inhibits CRISPR-Cas9 engineering in human pluripotent stem cells. Nat Med. 2018;1:939-46. https://doi. org/10.1038/s41591-018-0050-6.

Kleinstiver BP, Pattanayak V, Prew MS, Tsai SQ, Nguyen NT, Zheng Z, et al. High-fidelity CRISPR-Cas9 nucleases with no detectable genome-wide off-target effects. Nature. 2016;529:490-5. https://doi.org/10.1038/nature16526.

Kosicki M, Tomberg K, Bradley A. Repair of double-strand breaks induced by CRISPR-Cas9 leads to large deletions and complex rearrangements. Nat Biotechnol. 2018;36:765. https://doi.org/10.1038/nbt.4192.

Organizing Committee for the International Summit on Human Gene Editing, 2015. On human gene editing: international summit statement [WWW document]. URL http://www8. nationalacademies.org/onpinews/newsitem.aspx? RecordID=12032015a (accessed 6.30.18). 
Schaefer KA, Wu W-H, Colgan DF, Tsang SH, Bassuk AG, Mahajan VB. Unexpected mutations after CRISPR-Cas9 editing in vivo. Nat Methods. 2017;14:547-8. https://doi. org/10.1038/nmeth.4293.

Shmakov S, Smargon A, Scott D, Cox D, Pyzocha N, Yan W, et al. Diversity and evolution of class 2 CRISPR-Cas systems. Nat Rev Microbiol. 2017;15:169-82. https://doi.org/10.1038 /nrmicro.2016.184.

Swarts DC, van der Oost J, Jinek M. Structural basis for guide RNA processing and seed-dependent DNA targeting by CRISPR-Cas12a. Mol Cell. 2017;66:221-233.e4. https://doi.org/10.1016/j.molcel.2017.03.016.

Wang, D.C., Wang, X. Off-target genome editing: a new discipline of gene science and a new class medicine. Cell Biol Toxicol. 2019. doi: https://doi.org/10.1007 /s10565-019-09475-7.
Wang W, Zhang L, Wang X, Zeng Y. The advances in CRISPR technology and 3D genome. Semin Cell Dev Biol. 2018;90: 54-61. https://doi.org/10.1016/J.SEMCDB.2018.07.009.

Yamano T, Nishimasu H, Zetsche B, Hirano H, Slaymaker IM, Li $\mathrm{Y}$, et al. Crystal structure of Cpf1 in complex with guide RNA and target DNA. Cell. 2016;165:949-62. https://doi. org/10.1016/J.CELL.2016.04.003.

Zetsche B, Gootenberg JS, Abudayyeh OO, Slaymaker IM, Makarova KS, Essletzbichler P, et al. Cpfl is a single RNA-guided endonuclease of a class 2 CRISPR-Cas system. Cell. 2015;163:759-71. https://doi.org/10.1016/j. cell.2015.09.038.

Publisher's note Springer Nature remains neutral with regard to jurisdictional claims in published maps and institutional affiliations. 